\title{
Interactive Design of Bus Station Suitable for Aging Based on Sensory Enhancement
}

\author{
Shu YANG ${ }^{1}, \mathrm{Yi}_{\mathrm{WANG}}{ }^{*}$, Yi Xiao XUAN ${ }^{1}$ \\ ${ }^{1} \mathrm{Xi}$ 'an University of Technology, Shanxi, Xi'an, 710054
}

\begin{abstract}
Aiming at the problem of the sensory function decline in the elderly, the research intends to improve the optimal aging of bus stops by sensory enhancement interaction design. Though observing the behavior of the elderly and combining the user's journey map and the KANO model to explore the elderly's waiting needs at the bus station, it is concluded that the use of visual amplification, voice prompts, handrail dependence and other aspects of sensory enhancement can well improve the interactive design of bus station suitable for the elderly with weak senses, solve the problem of poor information interaction between the elderly and the bus station, and provide ideas for the aging intelligent bus system in the future.
\end{abstract}

\section{Introduction}

Under the service philosophy of accommodating the elderly and based on old people, the interaction between the elderly and the product is designed more through the sensory system of the human body. Reference 1 uses a sensory compensation mechanism, which cuts in from three perspectives of the senior citizen's physiological characteristics, behavioral interaction, and emotional experience, and analyzes the interaction problems between the elderly and smart products ${ }^{[1]}$. However, for the elderly with different degrees of deterioration in all senses, sensory compensation is difficult to play an effective role. Therefore, on this basis, the design method of sensory enhancement is more suitable for the senior citizens.

Relevant research that takes aging transportation service design as an entry point, combined with sociology, service science, behavior geography and other disciplines, provides a design dimension thinking method and direction for the existing aging service design of public transportation in cities $^{[2]}$. Reference 3 clearly pointed out that the information interaction between the elderly and the product is inaccurate, disfluency and uncoordinated ${ }^{[3]}$. This article focuses on the design and research of the interaction between the elderly and the bus station, and enhances the sensory design from three aspects: visual sense, auditory sense, and tactile sense, to optimize the interaction between the elderly and the bus station.

\section{Status Quo of Interactive Design for Bus Stations Suitable for Aging}

With the increase of age, the physical function and psychological aspects of the elderly have declined in varying degrees, such as vision loss, hearing loss, slow response, weakened limb coordination, and reduced sensitivity to the outside world. Meanwhile, some elderly people are also accompanied by various problems such as diseases and disabilities. From the perspective of the psychological needs of the elderly whose demand for socialization is gradually strong, and they also have sufficient free time, therefore the frequency of travel of the elderly has increased. The interaction between the elderly and the bus station becomes particularly important.

In the research on the current situation of the aging interactive design of bus stations, field investigation are mainly used to conduct behavior observation and questionnaire interviews with the elderly. Among the elderly people interviewed, most of them are between the ages of 65 and 75, and travel time period is usually from 8 am to 9 am. Most of the elderly people in this time period take buses to parks and other places for exercise, and some elderly people pick up and drop off children go to school.

In the interviews with the elderly, regarding the interactive design of the bus stations currently in use, most of the elderly believe that it is very convenient to use the elderly bus card to take the bus for free due to a series of public welfare policies that take the bus. Many elderly people choose to travel by bus for a major reason.

During the interview, it was also found that there are still many problems in the interaction between the elderly and the bus station. When the elderly are waiting at the bus station, it is difficult to solve the problems when the vehicle arrives at the station and whether they can choose to transfer to other vehicles in time. In order to solve these problems, bus companies have launched various related WeChat applets or Apps to improve, but nowadays the elderly are still jerky and avoid using smart products, and it is difficult to learn to operate. 
Secondly, when the bus enters the station, the vehicle itself will broadcast the voice message that the bus has arrived at the station, but it is difficult for the elderly to receive prompt information clearly and accurately due to the loss of vision, hearing, slow response, and the surrounding environment. Finally, in the long-term waiting process of the elderly, some bus stations do not have parking or seats. Although some stations have seats and other facilities, due to their height problems and the seats themselves do not have armrests, the body reactions are not sensitive. The elderly are more difficult to implement.
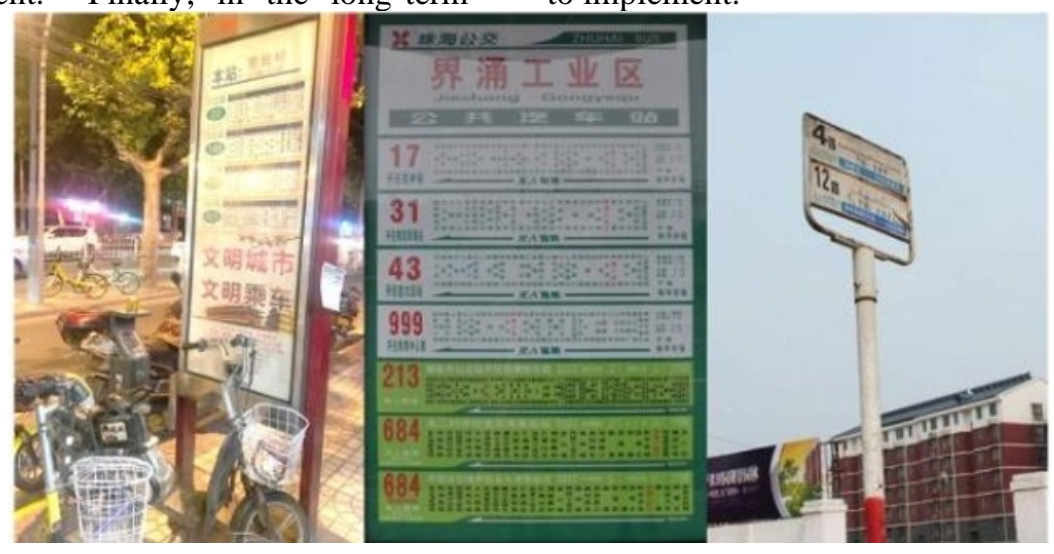

Fig. 1. Current Situation of Bus Station

\section{An Analysis of User Demands for Interchangeable Design of Bus Station}

\subsection{Analysis of Behavior Characteristics of the Elderly and User Journey Map}

When entering the elderly stage, their leisure behaviors, life behaviors, shopping behaviors, etc. are changing. A comprehensive understanding of the behavioral characteristics of the elderly will help to better understand the needs of the elderly. The daily behavioral characteristics of the elderly are closely related to their living environment, physical condition, economic conditions, hobbies, etc., and to a certain extent, they are jointly affected by psychology and physiology.

Compared with other stages of behavior, the behavior of the elderly has the characteristics of regularity, aggregation and habituation. By understanding the behavioral characteristics of the elderly, it helps to better understand the needs of the elderly and improve the pleasantness and applicability of the elderly products.
Understand the behaviors of the elderly and apply them to the design of service robots for the elderly to provide a more comfortable and user-friendly design for the elderly and truly focus on "people".

In the analysis of the current situation of the aging interactive design of the bus station, we observe the behaviors of the elderly. According to a series of behaviors of the elderly in the bus station, a user journey map of the elderly user is drawn to accurately grasp the pain points and opportunities in the interaction process of the elderly in the bus station, as well as their overall emotional changes.

The user journey map is mainly divided into three stages: entering the station, obtaining station information, and waiting process. By deconstructing the behavioral activities and emotional changes of the elderly in the bus stations in these three stages, it can be clearly seen from the figure that the lowest point of emotions appears in the waiting process. During the waiting process, the elderly's emotional fluctuations also change more. So we can analyze the pain points of the elderly users here and address their pain points. The problem is the opportunity for designers to optimize their designs.

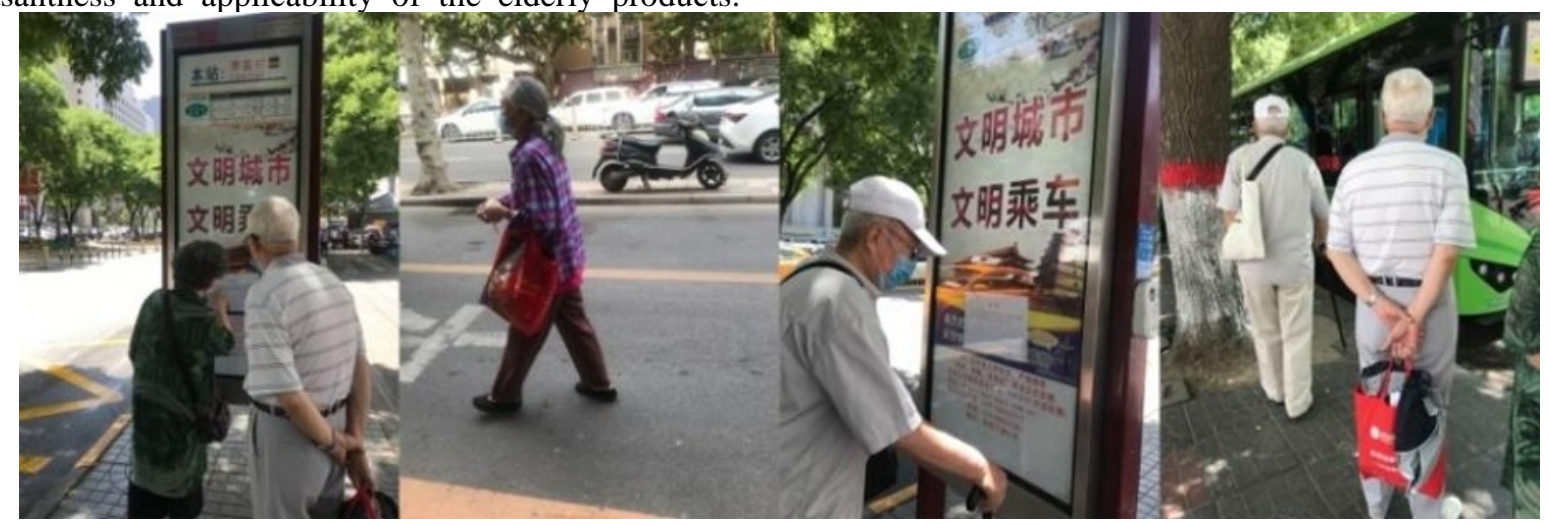

Fig. 2. Behavior Observation of the Elderly in Bus Stations 


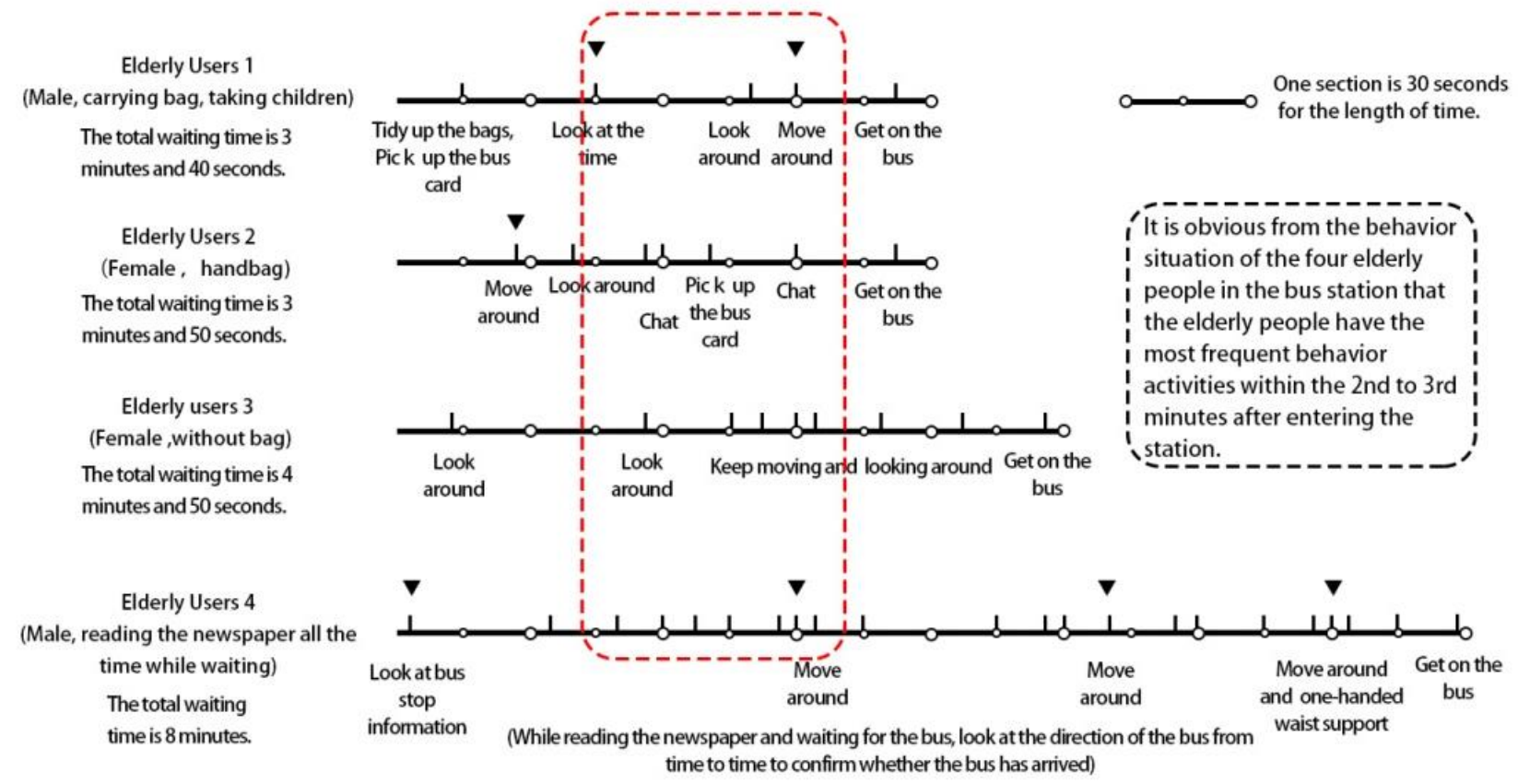

Fig. 3. Situation Chart of Bus Stations for the Elderly

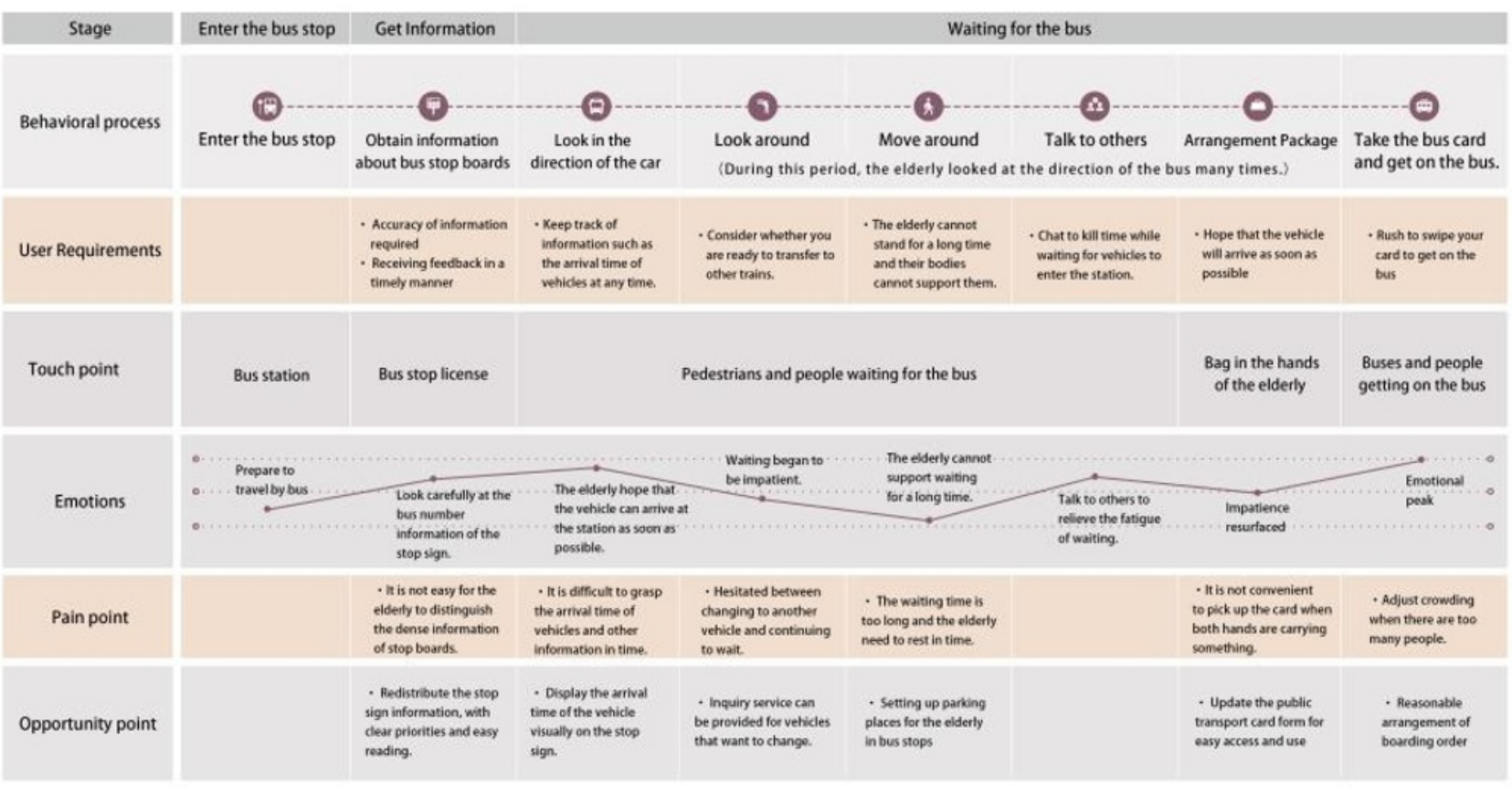

Fig. 4. User journey map

\subsection{User demand analysis based on KANO model}

The KANO model, invented by Professor Kano Koji, Tokyo Institute of Technology, is a useful tool for classifying and prioritizing user needs, and it can intuitively analyze the priority of user needs. According to the relationship between different types of quality characteristics and customer satisfaction, Professor Kano divides it into five categories: basic demand, expectation demand, excitement demand, indifferent demand and reverse demand ${ }^{[4]}$.

The first level is the basic demand, that is, the necessary functions or attributes that the product itself must have. For all user groups, the most basic and main function that a bus station should possess is the complete function of the station sign information and station stops and other physical facilities. 
The second level is the expected demand, that is, the functional requirements that users expect from the product. The problem that the aging interactive design of the bus station is expected to solve is to make the interaction between the bus station and the elderly more natural and convenient, so that the elderly can receive the information of the bus station in time and give effective feedback. Demand at this level is also a pain point for users in product design.

The third level is the excitement demand, also known as the charm demand, that is, the need for icing on the cake. The designer can plan and design the bus station to provide the elderly with some unexpected services in addition to basic needs when interacting with the elderly group, so that the elderly group can enjoy a better quality experience $^{[5]}$.

The fourth level is the indifferent demand, that is, the requirements that have no impact on user experience satisfaction regardless of whether they are provided or not. For the elderly, the design of billboards in bus stations belongs to this level of demand. The existence of itself does not affect the interactive experience between the elderly and the bus station.

The fifth level is the reverse demand, that is, users do not have this demand, and increasing this demand will instead reduce the user's experience.

Table 1. Age-appropriate interaction of bus stations User requirements

\begin{tabular}{c|ll}
\hline hierarchy of demand & Demand category & \multicolumn{1}{c}{ Demand content } \\
\hline first & basic demand & $\begin{array}{l}\text { 1. Complete and accurate information of the bus schedules } \\
\text { 2. Other complete facilities in the bus station }\end{array}$ \\
\hline second & expected demand & $\begin{array}{l}\text { 1. Correspondence between the information of the stop sign and the } \\
\text { physical habits of the elderly } \\
\text { 2. Flexible operation by the elderly } \\
\text { 3. Easy information collection and feedback for and from the elderly } \\
\text { 4. Timely information reception such as changing stations }\end{array}$ \\
\hline \multirow{2}{*}{ fourth } & excitement demand & $\begin{array}{l}\text { 1. Real-time grasp of the bus information you need } \\
\text { 2. Exclusive waiting place for the elderly }\end{array}$ \\
& indifferent demand & $\begin{array}{l}\text { 1. Complete facilities in the bus station, such as billboards, trash cans, } \\
\text { etc. } \\
\text { 2. Complete and accurate information shown on the stop sign }\end{array}$ \\
\hline
\end{tabular}

In this process, the designer first needs to ensure that the user's basic needs are met and the basic functional attributes of the product are met to solve the user's most basic functional needs. Secondly, the designer should try his best to meet the user's expectations according to the user survey. This level of demand is the main battlefield that differentiates it from other similar products. The satisfaction of the expected demand can greatly improve the market competitiveness of the product and user satisfaction. The designer analyzes the user's pain points and itching points, and performs functional design or supplementation on the problems between the product and the user, so as to improve the integrity of the current product and increase user satisfaction. Finally, the designer can further increase the user's sense of identity and belonging to the product by conceiving some small additional design points that are unexpected to the users.

\section{Sensory-enhanced interactive design of bus station suitable for aging}

\subsection{Sensory enhancement}

Sensory enhancement is to amplify a certain sensory system to enable users to receive information more effectively and accurately and make necessary feedback in a timely manner with the help of technology and equipment. This article starts with the realization of multisensory enhancement, and designs the multisensory interaction between the bus station and the user, so that the elderly can still communicate with bus station the same way as healthy adults when their physiological functions decline and their senses weaken. Stations interact normally, making it easier for the elderly to travel by bus.

The sensory compensation mechanism refers to that when the perception ability of a certain sense declines or is damaged, another kind of sensory compensation that normally receives information is enhanced to ensure the integrity and accuracy of information reception, which is essentially a compensation mechanism. However, due to the decline of the overall physiological functions of the elderly, various sensory functions have various degrees of weakness or even imperfection. The sensory compensation mechanism of the elderly group cannot 
generally function effectively with healthy adults. Only by externally strengthening the sensory system to make the sensory function of the elderly reach the level of the normal young and middle-aged population, can the elderly more easily and quickly perceive the external information. Therefore, this paper adopts the method of sensory enhancement to carry out the aging interactive design of bus stations.

For example, a nail clipper specially designed for the elderly has been launched on the Internet. It is different from the ordinary nail clippers. A small magnifying glass is added to the position of the knife head. Aiming at the problem of the general decline of visual function and presbyopia in the elderly, the magnifying effect of the convex lens on the vision is used to enhance the visual sensory experience, so that the elderly group can still use the nail clipper as normal people in the case of vision decline or normal.

\subsection{Aging interactive design of bus station based on sensory enhancement}

\subsubsection{Visual sensory enhancement}

Vision is one of the most direct ways for people to receive outside information. By visually strengthening the elderly's perception of interactive information, the purpose of effectively receiving outside information is achieved. Designers can block design the primary and secondary relationship of the information displayed on the interface of the bus station sign, using different colors for visual emphasis. While using different colors for emphasis, also pay attention to the number of colors used and the choice of color system to avoid secondary difficulties for the elderly to read the content. For the problem that the elderly cannot see the information on the interface of the stop sign clearly, the designer can enhance the visual sense through visual enlargement. For example, a magnifying glass that is operated up and down by a pulley on the bus stop sign is provided to facilitate the use of the elderly. sign interface, different dynamic effect changes can be adopted and appropriate transformation speeds can be adopted to improve the reading ability of the elderly.

\subsubsection{Auditory sensory enhancement}

Hearing is the widest way to spread and receive information. Designers can guide the behavior of the elderly during the ride by enhancing auditory senses. Voice announcement and voice prompt function are used to prompt the real-time information of the elderly bus trip and remind them of vehicle arrival. For the problem of obtaining information in the station and bus travel information, a voice interaction function can be added to guide the elderly to operate, instead of a simple text description form. Voice interaction intuitively and effectively conveys information to the elderly, and greatly liberates the use of the elderly's hands and eyes, eases the physical burden of the elderly, and at the same time, voice interaction gives anthropomorphic emotions, which can bring the elderly more comfortable and warm experience.

\subsubsection{Tactile sensory enhancement}

There are many sensory cells connected to the sensory system on the human skin to help receive various stimulus signals transmitted to us by the external environment. People can give more sensitive feedback to the information transmitted by the tactile sensory system. Designers can add interactive design that enhances the sense of touch in the design of suitable aging bus stations, such as sensory enhancement design in the form of vibration, and can add a vibration function to the armrest or backrest of the waiting seat of the bus station to become a bus. It will serve as a warning to the elderly when they are about to arrive. In addition, the armrest can be provided to allow the elderly to take a short rest during the long-term waiting process. The rough particle surface is used in the armrest to enhance the tactile sense and facilitate the elderly to grasp the armrest.

\subsection{Multisensory integration and interactive design}

When we are looking for friends in a noisy crowd, it is easier for us to discover when the other side waves and yells. In order to complete the search task faster, information from different senses (visual: waving arms; auditory: shouting) not only interact when they are input at the same time, but also merge into a coherent and meaningful expression. When multisensory stimuli occur simultaneously, the interaction between various sensory systems and the process of achieving a certain fusion with each other are called multisensory association.

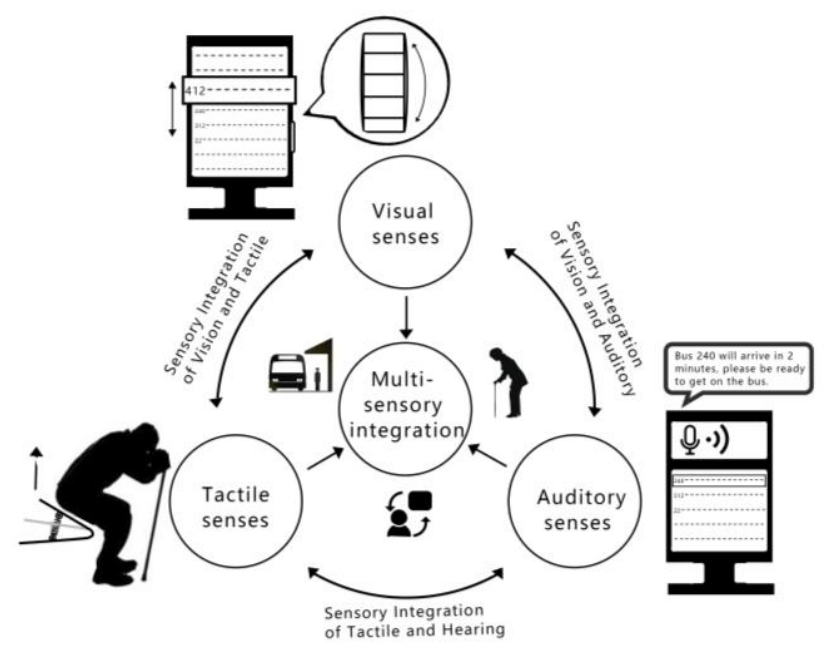

Fig. 5. Multisensory integration interaction design

The decline in the physiological function of the elderly causes different declines in various senses. Some elderly people may even lose one or more senses after old age. Unilateral sensory enhancement has not been very effective for this part of the elderly function, when the information formed by a single sense is unreliable or 
completely unavailable, the necessity of integrating information from multiple senses to make the best use of all available inputs is significantly increased. Even studies in the past few decades have shown that when a certain sensory ability is lacking or declining, it is more conducive to the union of multiple senses. Therefore, designers should continuously cut into the aging interactive design from the perspective of multisensory union. Reference 1 also stated that sensory association can effectively avoid the physical burden caused by a single perception method to the elderly, and the combination of the sensory capabilities of the human senses can greatly expand the interaction space between the product and the elderly to achieve more optimization interactive experience.

For example, a blood glucose tester designed for the elderly, uses a contrasting color to divide the overall interface of the tester into two parts. The functional area is concise and clear, so that the elderly can see the visual sense at a glance, and the test results are displayed on the screen of the tester. Broadcast in the form of voice to realize the combination of visual and auditory senses; in the case of danger, the voice reminder is accompanied by the overall sense of vibration of the fuselage to realize the combination of auditory and tactile senses.

The advancement of technology development has gradually entered the lives of people along with the convenience of the Internet information era, intelligent life, and smart old age. The technology-assisted multisensory jointing interactive design is also suitable for the aging interactive design of bus stations.

Set up special seats or backrests for the elderly at the bus station. The armrests and backrests of the seats are equipped with vibration functions to transmit information to users through tactile sensory stimulation; LED screens are used to display stop sign information (including line stations, real-time positions of vehicles and estimated time of arrival) and use different colors of text to distinguish the driving information of vehicles that are coming to the station and those who are not arriving, to achieve enhanced visual sensory stimulation; in addition, an artificial voice intelligent system needs to be added to provide intelligent voice answers to the questions raised by the elderly. It can broadcast the current vehicle position in time and accurately report the estimated arrival time. When the waiting vehicle is about to arrive, it will warn the user through the joint stimulation of three senses: tactile, visual, and auditory, so that the elderly can receive information more accurately and make preparations for getting up and getting on the vehicle promptly, and it is more effective to reduce the visibility. It can reduce the error rate of the vehicle number, and guide the elderly to better interact with the bus station. At the same time, the anthropomorphic artificial intelligence voice can enhance the intimacy of the bus station and the user's sense of security, and more naturally eliminate the fear of the elderly for strange products.

\section{Concluding remarks}

With the advent of the era of smart life, designers have more space for aging interactive design directions. Based on the interaction between the bus station and the elderly, this paper adopts the design method of sensory enhancement, and proposes a sensory enhancement method based on visual enhancement, auditory enhancement, tactile enhancement, as well as artificial intelligence and augmented reality technology. Based on the combination of scientific and technological suitable aging interactive design methods, the new interactive design ideas for the bus station are served, and a more clear direction is proposed in the corresponding aspect, so as to solve the problem between the elderly and the bus station. The contradictory problems generated by interaction can achieve the purpose of suitable aging design. The focus of follow-up is to organically combine the above contents from the technical aspects, and present a complete interactive design of suitable aging bus stations.

\section{Acknowledgement}

This research was financially supported by the National Social Science Fund of China(Grant No.18BG132)

\section{About the Author}

Wang Yi (1973-), female, from Zhaoyuan, Shandong Province, doctor, professor and master's tutor of the School of Art and Design of Xi'an University of Technology, research interests: human-computer interaction design research, design archaeology theory research.710054,China

\section{References}

1. ZHANG Ping, DING Xiaomin. Research on Interaction Design of Intelligent Products for the Elderly Under Compensatory Mechanism[J].2018

2. GAN Wei, HU Fei. Research on the Design of Urban Existing Public Transportation Service Suitable for Aging[J]. 2017

3. ZHOU Ming, Li Ya-Jun. Interative Service Design for the Aged-in China[J]. 2017

4. HE Xue Feng. Hierarchical Analysis of Product Requirements Based on KANO Model[J]. 2014

5. Product Designers Must Understand Demand Analysis-KANO Model [EB/OL]. Https://www.sohu.com/a/362094988_505826. 2019$12-22$. 